\title{
A mathematical model reveals the influence of NPIs and vaccination on SARS-CoV-2 Omicron Variant
}

\section{Zhi-Cheng Wang \\ Lanzhou University \\ Yan Wu \\ Lanzhou University \\ Yongping Xiong \\ Lanzhou University \\ Jiangqian Zhang \\ Lanzhou University \\ Zhihui Ma \\ Lanzhou University}

Bin-Guo Wang ( $\nabla$ wangbinguo@lzu.edu.cn )

Lanzhou University https://orcid.org/0000-0002-7991-6665

\section{Research Article}

Keywords: SVEIR Omicron model, Reproduction numbers, NPIs, Vaccines, Sensitivity analysis, Herd immunity

Posted Date: February 21st, 2022

DOI: https://doi.org/10.21203/rs.3.rs-1324280/v1

License: (c) (i) This work is licensed under a Creative Commons Attribution 4.0 International License.

Read Full License 


\title{
A mathematical model reveals the influence of NPIs and vaccination on SARS-CoV-2 Omicron Variant
}

\author{
Bin-Guo Wang, Zhi-Cheng Wang, Yan Wu, Yongping Xiong, Jiangqian Zhang \\ and Zhuihui Ma \\ School of Mathematics and Statistics, Lanzhou University, \\ Lanzhou, Gansu 730000, People's Republic of China.
}

\begin{abstract}
A SVEIR SARS-CoV-2 Omicron variant model is proposed to provide some insight to coordinate non-pharmaceutical interventions(NPIs) and vaccination. Mathematically, we define the basic reproduction number $\mathscr{R}_{0}$ and the effective reproduction number $\mathscr{R}_{e}$ to measure the infection potential of Omicron variant and formulate a optimal disease control strategy. Our inversion results imply that the sick period of Omicron variant in the United States is longer than that of Delta variant in Indian; The decreasing of the infectious period of the infection with infectiousness implies that the risk of hospitalization is reduced; but the increasing period of the infection with non-infectiousness signifies that Omicron variant lengthens the period of nucleic acid test being negative; Optimistically, Omicron's death rate is only a quarter of Delta's. Moreover, we forecast that the cumulative cases will exceed 100 million in the United States on 28 February, 2022 and the daily confirmed cases will reach a peak on 2 February, 2022. The results of parameters sensitivity analysis imply that NPIs is helpful to reduce the number of confirmed cases. Especially, NPIs are indispensable even if all the people were vaccinated when the efficiency of vaccine is relatively low. By simulating the relation ships of the effective reproduction number $\mathscr{R}_{e}$, the vaccination rate and the efficacy of vaccine, we find that it is impossible to achieve the herd immunity without NPIs while the efficiency of vaccine is lower than $88.7 \%$. Therefore, the herd immunity area is defined by the evolution of relationships between the vaccination rate and the efficacy of vaccine. Finally, we present that the disease-induced mortality rate demonstrates the periodic oscillation and an almost periodic function is deduced to match the curve. A discussion completes the paper.
\end{abstract}

Keywords: SVEIR Omicron model; Reproduction numbers; NPIs; Vaccines; Sensitivity analysis; Herd immunity

AMS Subject Classification (2020): 34A34; 34D20; 92D30

*Corresponding author (wangbinguo@lzu.edu.cn) 


\section{Introduction}

Coronavirus disease 2019 (COVID-19), caused by a novel virus of the coronavirus genus (SARSCoV-2), has lasted for more than two years and caused a Once-in-a-Century global crisis [47]. Despite scientists worldwide racing to develop antiviral drugs, curative treatments are unavailable at the time of writing, and vaccinations against the COVID-19 has been recently distributed around the world, the global economy is experiencing the worst plunge in recent history amid fears of further deterioration of the COVID-19 situation [13]. Since the outbreak of COVID-19 was first detected in December 2019 in Wuhan, China [22], many authors have studied the transmission dynamics of COVID-19 by various means and methods $[3,7,12,17,21,31,36,44-46]$.

As same as all viruses, SARS-CoV-2 which causes COVID-19 pandemic changes over time. For those changes, most of them do not impact on the virus's properties. However, there may be some changes that have an important impact on the characteristics of the virus, such as how easily it is to spread, the severity of the associated diseases, the performance of vaccines and therapeutic medicines, the applications of the diagnostic tools, and/or the other public health and control measures [48]. For example, the B.1.1.529 (Omicron) variant, which was first identified in South Africa in early November 2021 [5], has rapidly become the dominant variant in many countries. The published research on live-virus neutralization assays revealed that Omicron could be escape antibody neutralization by the BNT162b2 messenger RNA vaccine (PfizerioNTech) [29].

Generally, population-wide rapid nucleic acid testing, isolation, sterilizing, and social distancing, which are called NPIs(non-pharmaceutical interventions), have played an important role to prevent and control the transmission of SARS-CoV-2 [6]. For example, many countries have been enforcing NPIs, such as social distancing (also called contact restrictions) and travel restrictions, to control the development of the SARS-CoV-2 [18,19]. Tian et al. [37] showed that the confirmed COVID-19 cases outside Wuhan would have decreased to 744,000 $( \pm 156,000)$ due to the Wuhan travel ban or the national emergency response. Pavelka et al. [26] have investigated the influence of population-wide rapid antigen testing on SARS-CoV-2 prevalence in Slovakia. Their result$\mathrm{s}$ showed that the prevalence decrease was not solely contributed by infection control measures, while the addition measures, such as the isolation and quarantine of household members of those testing positivity, were also required. Since SARS-CoV-2 is transmitted by droplets and aerosols, Cheng et al. [8] showed that the surgical masks were effective on preventing virus spread under conditions of low virus abundance (virus-limited). However, more advanced masks and other protective equipments were required in potentially virus-rich indoor environments, including medical centers and hospitals. Senapati et al. [34] revealed that it is necessary to take a higher intervention effort to control the disease outbreak within a shorter period of time in India. Further researches which has been proposed for considering the impact of NPIs on the spread of SARS-CoV-2 could be found in $[20,27,28,33,38,41,43]$.

In fact, an excessive NPIs has restricted the development of the global economies and impacted on the general quality of life (in particular, mental health) [24]. That's a general public perception that vaccines are the most effective defense to control the disease completely. Saad-Roy et al. [35] explored three scenarios of selection and found that a one-dose policy may increase the potential for antigenic evolution under certain conditions of partial population immunity. Moreover, they 
highlighted the critical need to test viral loads and quantify immune responses after one vaccine dose, and to ramp up vaccination efforts throughout the world. In consideration of limited initial supply of SARS-CoV-2 vaccine, Bubar et al. [3] used a mathematical model to compare five agestratified prioritization strategies. Following some of the WHO-SAGE recommendations, AcuñaZegarraa et al. [2] formulated an optimal control problem with mixed constraints to describe vaccination schedules.

Since someone was diagnosed with the Omicron variant of COVID-19 despite having received two shots of the vaccine, NPIs remain very indispensable to terminate the pandemic of SARS$\mathrm{CoV}-2$. Hence, it is necessary to identify strategies for safely relaxing nonpharmaceutical measures $[16,25]$. Drawing support from optimization-based control on an age-differentiated compartmental model, Grundel et al. [14] studied the relations of vaccination and social distancing. However, the published which coordinated NPIs and vaccination comprehensively to prevent the outbreak of Omicron variant is less common. In this paper, we propose a SVEIR SARS-CoV-2 Omicron variant model to reveal the influence of NPIs and vaccination on SARS-CoV-2 Omicron variant in four key aspects: (1)Mathematically, we define the basic reproduction number $\mathscr{R}_{0}$ and the effective reproduction number $\mathscr{R}_{e}$ to measure the infection potential of Omicron variant and develop disease control strategies. (2)Parameter inversion is conduted to explore the mechanism of Omicron variant and give some suggestions to stay home and isolate from other people. (3)Sensitivity analysis find the main factors affecting the spread of Omicron variant, and formulate prevention and control strategies. (4)Facing a low vaccination willingness and efficacy of vaccines, we explore the herd immunity area.

The remainder of this paper is structured as follows. We present the compartmental model in section 2 and describe the extinction and uniform persistence in section 3 . Section 4 is dedicated to the case study, and the paper is concluded in section 5 .

\section{A compartmental model with NPIs and vaccination.}

A SEIR model has been used to study the transmission of COVID-19 [30,42]. Furthermore, in this paper, each of the exposed and infectious class is further divided into two groups, one contagious and other non-contagious [17]. Consider the transmission of Omicron variant with NPIs and vaccines, the population is divided into the following categories: Let $S$ be the number of susceptible individuals, $V$ be the number of the vaccinated individuals, $E_{1}$ be the number of the exposed individuals who are not contagious in the early stages, $E_{2}$ be the number of the exposed individuals who can infect the susceptible, and $I_{1}$ be the number of the infectious individuals who are contagious, $I_{2}$ be the number of the infectious individuals who are not contagious, $R$ be the number of recovery individuals, $N$ be the total population, that is, $N=S+V+E_{1}+E_{2}+I_{1}+I_{2}+R$. Furthermore, we suppose that $N$ is constant. Refer to [17], the population growth process can be described as in Figure 1. 


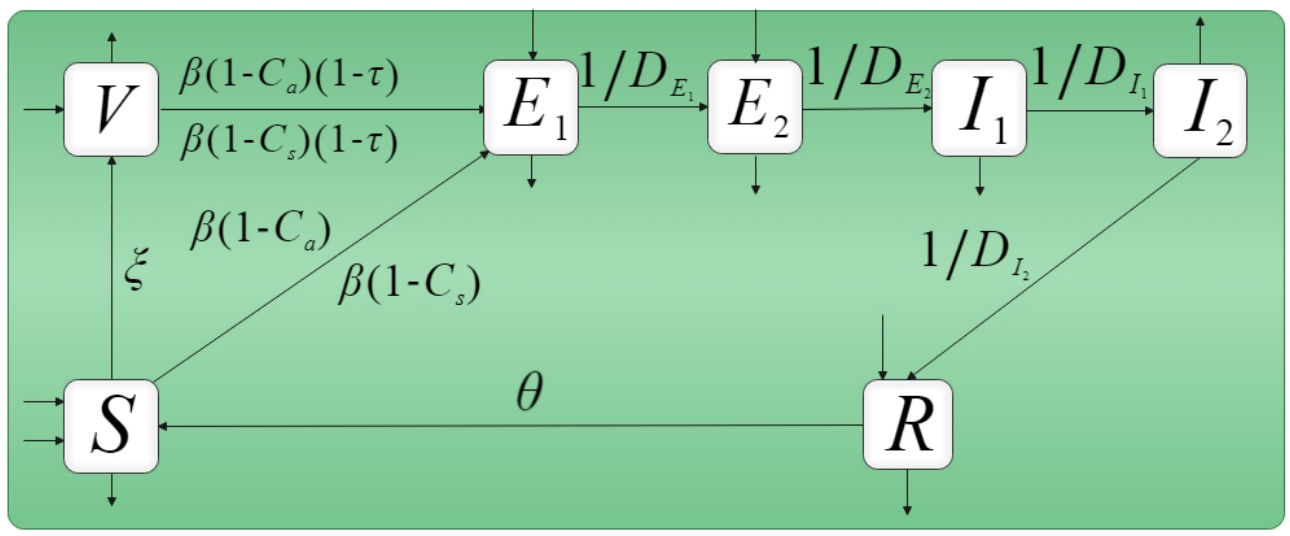

Figure 1: Compartmental diagram of COVID-19 transmission dynamics

The model is given by an autonomous system of ordinary differential equations

$$
\begin{aligned}
& \frac{d S}{d t}=\Lambda-\beta\left(1-C_{a}\right) \frac{S E_{2}}{N}-\beta\left(1-C_{s}\right) \frac{S I_{1}}{N}-\xi S-\mu S+\theta R, \\
& \frac{d V}{d t}=\xi S-\beta\left(1-C_{a}\right)(1-\tau) \frac{V E_{2}}{N}-\beta\left(1-C_{s}\right)(1-\tau) \frac{V I_{1}}{N}-\mu V \text {, } \\
& \frac{d E_{1}}{d t}=\beta\left(1-C_{a}\right) \frac{S E_{2}}{N}+\beta\left(1-C_{s}\right) \frac{S I_{1}}{N}+\beta\left(1-C_{a}\right)(1-\tau) \frac{V E_{2}}{N}+\beta\left(1-C_{s}\right)(1-\tau) \frac{V I_{1}}{N} \\
& -\frac{E_{1}}{D_{E_{1}}}-\mu E_{1} \\
& \frac{d E_{2}}{d t}=\frac{E_{1}}{D_{E_{1}}}-\frac{E_{2}}{D_{E_{2}}}-\mu E_{2}-d E_{2}, \\
& \frac{d I_{1}}{d t}=\frac{E_{2}}{D_{E_{2}}}-\frac{I_{1}}{D_{I_{1}}}-\mu I_{1}-d I_{1}, \\
& \frac{d I_{2}}{d t}=\frac{I_{1}}{D_{I_{1}}}-\frac{I_{2}}{D_{I_{2}}}-\mu I_{2}, \\
& \frac{d R}{d t}=\frac{I_{2}}{D_{I_{2}}}-\mu R-\theta R,
\end{aligned}
$$

where $\Lambda$ is the recruitment rate of susceptible class, $C_{a}$ and $C_{s}$ denote the intensity of NPIs for the individuals of the exposed individuals and the infectious individuals who can infect the susceptible, respectively. $\beta$ denotes the effective contact rate, $\xi$ denotes the vaccination coverage rate, $\theta$ is the antibody disappear rate of recovery class, $\mu$ is the natural death rate of the population, $D_{E_{1}}$ and $D_{E_{2}}$ are lengths of the incubation with non-infectiousness and incubation with infectiousness, respectively. $0 \leq \tau \leq 1$ denotes the vaccine efficacy $(\tau=1$ represents a vaccine that offers $100 \%$ protection against infection, $\tau=0$ models a vaccine that offers no protection at all). $D_{I_{1}}$ and $D_{I_{2}}$ are lengths of the infection with infectiousness and infection with non-infectiousness, respectively. $d$ denotes the disease-induced mortality rate. Biologically, we could suppose that the number of total human population stabilizes at $N>0$.

For simplicity, set $\psi_{t}\left(x^{0}\right)$ be the solution of $(2.1)$ with initial value $\psi_{0}\left(x^{0}\right)=x^{0} \in \mathbb{R}_{+}^{7}$. By [11, Theorem 2.1], we have the following.

Theorem 2.1 For any $x^{0} \in \mathbb{R}_{+}^{7}$, system (2.1) has a unique nonnegative solution $\psi_{t}\left(x^{0}\right)$ with initial value $\psi_{0}\left(x^{0}\right)=x^{0}$, and all solutions are ultimately bounded and uniformly bounded. 
Remark 2.2 If we take $\tau=0$ or $\xi=0$ in (2.1), then the model consider that NPIs for the exposed and the infectious individuals who can infect the susceptible is the only measure. Similarly, let $C_{a}=C_{s}=0$ in the above discussion, system (2.1) implies that the vaccination is only gotten involved. If $\tau=\xi=C_{a}=C_{s}=0$, it means that there are no external factors involved (no vaccines, masks or other epidemic prevention measures). In this situation, the transmission dynamics of Omicron variant are studied based on the natural characteristics of Omicron variant itself.

\section{Reproduction number}

Reproduction numbers (ratio) are a crucial threshold parameter in the study of disease transmission. In epidemiology, it is defined as the expected number of secondary cases produced by a single (typical) infection in a completely susceptible population and is used to measure the infection potential of an infectious disease $[9,23]$. At the beginning of the transmission of coronavirus, based on likelihood and model analysis, Tang et al. $[3,7,31,36]$ revealed that the basic reproduction number may be as high as 6.47, which showed that COVID-19 is highly infectious. By means of the basic reproduction number, Bubar et al. [3] found a highly mitigated spread during vaccine rollout. Riley et al. [31] used a model of constant exponential growth and decay, and quantified this fall and rise in prevalence in terms of halving and doubling times and the basic reproduction number. Noting that an important quantity in epidemiological models, the basic reproduction number, Cuevas-Maraver et al. [7] discussed in the realm of the model what consequences different additional intervention measures would have had at the level of deaths and of cumulative infections. In this section, the definition and computation formulae of the basic reproduction number and the effective reproduction number for system (2.1) are established.

We first consider the disease-free solution of system (2.1). Let $E_{1}=E_{2}=I_{1}=I_{2}=0$, then we have

$$
\begin{aligned}
& \frac{d S}{d t}=\Lambda-\xi S-\mu S \\
& \frac{d V}{d t}=\xi S-\mu V
\end{aligned}
$$

By the similar arguments to those in [40], system (3.1) has a positive equilibrium $\left(S^{*}, V^{*}\right)=$ $\left(\frac{\Lambda}{\xi+\mu}, \frac{\xi \Lambda}{\mu(\xi+\mu)}\right)$, which is globally attractive. Linearizing system $(2.1)$ at the disease-free equilibrium $\left(S^{*}, V^{*}, 0,0,0,0,0\right)$, we get

$$
\begin{aligned}
\frac{d E_{1}}{d t}= & \beta\left(1-C_{a}\right) \frac{S^{*} E_{2}}{N}+\beta\left(1-C_{s}\right) \frac{S^{*} I_{1}}{N}+\beta\left(1-C_{a}\right)(1-\tau) \frac{V^{*} E_{2}}{N}+\beta\left(1-C_{s}\right)(1-\tau) \frac{V^{*} I_{1}}{N} \\
& \quad-\frac{E_{1}}{D_{E_{1}}}-\mu E_{1}, \\
\frac{d E_{2}}{d t}= & \frac{E_{1}}{D_{E_{1}}}-\frac{E_{2}}{D_{E_{2}}}-\mu E_{2}-d E_{2}, \\
\frac{d I_{1}}{d t}= & \frac{E_{2}}{D_{E_{2}}}-\frac{I_{1}}{D_{I_{1}}}-\mu I_{1}-d I_{1} .
\end{aligned}
$$

Let

$$
Y=\left(\begin{array}{ccc}
0 & F_{1} & F_{2} \\
0 & 0 & 0 \\
0 & 0 & 0
\end{array}\right), Z=\left(\begin{array}{ccc}
V_{1} & 0 & 0 \\
-V_{4} & V_{2} & 0 \\
0 & -V_{5} & V_{3}
\end{array}\right),
$$

where $F_{1}=\beta\left(1-C_{a}\right) \frac{S^{*}+(1-\tau) V^{*}}{N}, F_{2}=\beta\left(1-C_{s}\right) \frac{S^{*}+(1-\tau) V^{*}}{N}, V_{1}=\frac{1}{D_{E_{1}}}+\mu, V_{2}=\frac{1}{D_{E_{2}}}+\mu+d$, 


$$
\begin{gathered}
V_{3}=\frac{1}{D_{I_{1}}}+\mu+d, V_{4}=\frac{1}{D_{E_{1}}} \text { and } V_{5}=\frac{1}{D_{E_{2}}} \text {. Then we can rewritten (3.2) as } \\
\frac{d u}{d t}=(Y-Z) u .
\end{gathered}
$$

Motivated by the concept of next generation matrices introduced in $[9,23]$, we define the effective reproduction number of system (2.1) as

$$
\mathscr{R}_{e}:=\rho\left(Y Z^{-1}\right)
$$

where $\rho(A)$ denotes the spectral radius of a matrix $A$.

The following two theorems give a threshold-type result on the extinction and uniform persistence of the disease in terms of $\mathscr{R}_{e}$ in the natural state. The proof of the following two theorems can be seen in Appendix A.

Theorem 3.1 Assume $(A 1)-(A 2)$ holds and $\mathscr{R}_{e}<1$, then the disease-free equilibrium $\mathcal{E}^{*}=$ $\left(S^{*}, V^{*}, 0,0,0,0,0\right)$ of system $(2.1)$ is globally attractive.

Theorem 3.2 If $\mathscr{R}_{e}>1$, then there exists $\tilde{\varepsilon}>0$ such that the solution $\left(S(t), V(t), E_{1}(t), E_{2}(t), I_{1}(t)\right.$, $\left.I_{2}(t), R(t)\right)$ of system (2.1) with initial data $x^{0}$ in $\mathbb{R}_{+}^{7}$ and $\left(E_{1}(0), E_{2}(0), I_{1}(0)\right)>\hat{0}$ satisfies

$$
\liminf _{t \rightarrow \infty} E_{1}(t)>\tilde{\varepsilon}, \liminf _{t \rightarrow \infty} E_{2}(t)>\tilde{\varepsilon}, \liminf _{t \rightarrow \infty} I_{1}(t)>\tilde{\varepsilon} .
$$

Remark 3.3 If $\tau=0$ or $\xi=0$ hold in the above discussion, then we call (3.4) as the NPIs reproduction number, denoted by $\mathscr{R}_{e}^{N}$. Similarly, in the case of $C_{a}=C_{s}=0,(3.4)$ denotes the vaccines reproduction number $\mathscr{R}_{e}^{V}$. If $\tau=\xi=C_{a}=C_{s}=0$, then (3.4) is just the basic reproduction number $\mathscr{R}_{0}$. Furthermore, the conclusions of Theorem 3.1 and Theorem 3.2 remain available under different situations if we replace $\mathscr{R}_{e}$ by $\mathscr{R}_{e}^{N}, \mathscr{R}_{e}^{V}$ and $\mathscr{R}_{0}$.

\section{Numerical simulations}

Omicron variant is a new type of SARS-CoV-2. In this section, we take the data in the United States to explore the transmission mechanism and predict the development trend of Omicron.

\subsection{Parameters inversions}

We select the data in the United States showed in [49] from December 1, 2021 to January 30, 2022 , and use the fminsearch function in MATLAB to perform parametric inversion. The value of the vaccination coverage rate $\xi$ can be seen in [50]. In order to search the difference between Delta and Omicron variant, we compare the data in India [39] with that in the United States. The specific values are shown in Table 1 .

According to Table 1, we find that the Omicron variant highly infectious in the sense that $\beta=0.8993$ and the sick period of Omicron variant in the United States is 0.91 days longer than that of Delta variant in Indian; The decreasing of $D_{I_{1}}$ implies that the risk of hospitalization is reduced; but the increasing period of $D_{I_{2}}$ signifies that Omicron variant lengthens the period of 
nucleic acid test being negative; Optimistically, Omicron's death rate is only a quarter of Delta's. According to the value of $\theta$, we call for the four months interval of booster shot. Furthermore, if one who is an asymptomatic person has confirmed or suspected Omicron variant, regardless of the result of parameters inversion, should stay home and isolate from other people for at least 6 full days; the isolation time of symptomatic patients should be 3 days. Testing may be used to help determine when to end your isolation period.

Table 1: The parameters of disease in different regions

\begin{tabular}{ccccc}
\hline Parameters & Definitions & India & the United States & Unit \\
\hline$\beta$ & effective contact rate & 0.70 & 0.8993 & $/$ \\
$D_{E_{1}}$ & length of incubation with non-infectiousness & 2.9 & 3.07 & Days \\
$D_{E_{2}}$ & length of incubation with infectiousness & 2.3 & 1.51 & Days \\
$D_{I_{1}}$ & length of infection with infectiousness & 2.9 & 1.43 & Days \\
$D_{I_{2}}$ & length of infection with non-infectiousness & 12 & 15.00 & Days \\
$d$ & disease-induced mortality rate & $1.3 \times 10^{-4}$ & $2.97 \times 10^{-5}$ & Day $^{-1}$ \\
$C_{a}$ & NPIs for incubation with infectiousness & 0.20 & 0.1716 & $/$ \\
$C_{s}$ & NPIs for infection with infectiousness & 0.20 & 0.2991 & $/$ \\
$\mu$ & natural death rate of the population & $4 \times 10^{-5}$ & $2.44 \times 10^{-5}$ & Day \\
$N$ & total human population & 1380004000 & 120552473 & People \\
$\Lambda$ & recruitment rate of susceptible class & 65786 & 9877 & People/Day \\
$\theta$ & antibody disappear ratio of recover class & 0 & 0.0085 & $/$ \\
$\tau$ & vaccine efficacy & 0.85 & 0.3300 & $/$ \\
$\xi$ & vaccination coverage rate & 0.05 & 0.60 & $/$ \\
\hline
\end{tabular}

\subsection{Infection potential estimation}

By a simple calculation, the effective reproduction number of system (2.1) can be denoted that

$$
\mathscr{R}_{e}=\frac{\beta \Lambda(\mu+\xi(1-\tau))\left(D_{E_{2}}\left(d+\mu+\frac{1}{D_{I_{1}}}\right)\left(1-C_{a}\right)+1-C_{s}\right)}{\mu N D_{E_{1}} D_{E_{2}}(\xi+\mu)\left(\mu+\frac{1}{D_{E_{1}}}\right)\left(d+\mu+\frac{1}{D_{I_{1}}}\right)\left(d+\mu+\frac{1}{D_{E_{2}}}\right)} .
$$

It then follows from the inversion results in above subsection that $\mathscr{R}_{0}=8.883, \mathscr{R}_{e}^{N}=6.811$, $\mathscr{R}_{e}^{V}=5.952$ and $\mathscr{R}_{e}=4.564$. Therefore, it is shown that the infection potential of Omicron variant is very high without NPIS and vaccination in the United States. Even if one of NPIS and vaccination is implemented, Omicron variant might infect a large number of individuals. Obviously, the vaccination is better than that of NPIs for reducing the infectious potential of Omicron variant. Two countermeasures being implemented simultaneously remain the best way to protec$\mathrm{t}$ oneself from COVID-19 and reduce its impact on our communities. Furthermore, we analysis the correlation between parameters and the effective reproduction number $\mathscr{R}_{e}$ by random sample method with $\mathrm{N}=8000$, the result is shown in Figure 2. We can find however the parameters change, $\mathscr{R}_{e}$ mainly ranges $[3,5]$. This result reflects that the United States is facing a strong pressure for the prevention and control of Omicron. 


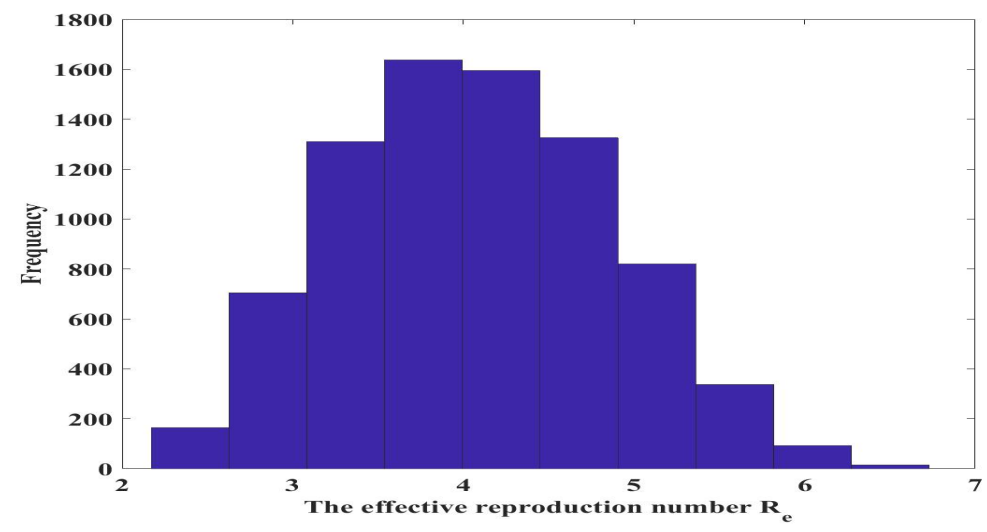

Figure 2: The peculiarity of $\mathscr{R}_{e}$

\section{3 $\quad$ Model fitting and tend predicting}

In the following, we forecast the spreading trend of Omicron pandemic in the United States. By a calculation, the numerical simulation results are shown in Figure 3.

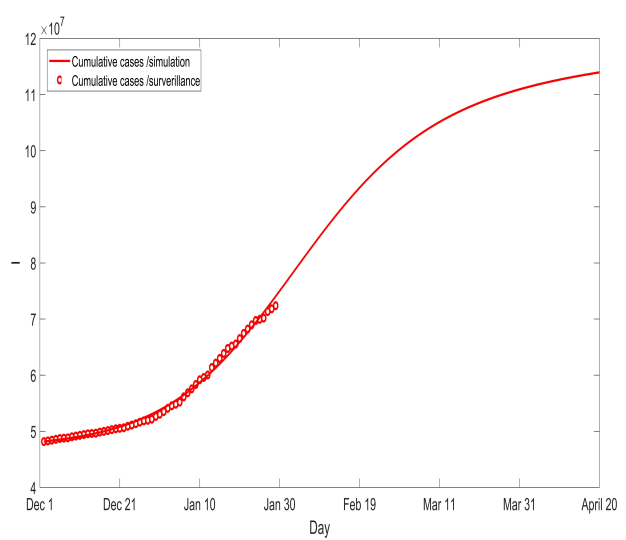

(a)

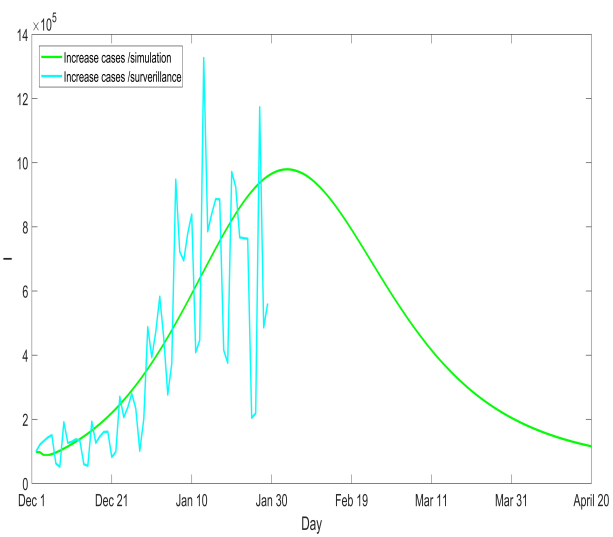

(b)

Figure 3: Numerical simulation of cumulative cases and increase cases

The discrete points and the blue line represent the real data of cumulative cases and increase cases from December 1, 2021 to January 30, 2022 in the United States, respectively. The red line and green line represent the simulation of cumulative cases and increase cases, respectively. We found that the dynamical results fit well with the statistical data [49](see Figure 3). During December 1, 2021 to April 20, 2022, Figure 3 forecasts that daily comfirmed cases will maintain an upward trend and reach the maximum value 979360 on February 2, 2022. And the cumulative cases will more than 100 million on February 28, 2022. Since the period of the infection with infectiousness decreases, we think many people are treated at home. It leads to the fact that the 
number of reports is lower than the predicted. There is a huge problem that some of American without any face coverings and unwilling to take the vaccine. Furthermore, if the existing protection intensity and vaccine injection schedule are maintained, the numerical simulation forecasts the COVID-19 in the United States will uniformly exist and form an endemic disease. Thus, it is imminent to tighten NPIs and accelerate vaccine programmes.

\subsection{Sensitivity analysis}

In this part, we compute Partial Rank Correlation Coefficients (PRCC) to identify the key factors which affect the change of $\mathscr{R}_{e}$ and the total infectious cases. In our experiment, we set that the parameters have a significant effect when $p$-value $<0.01$. The results can be seen in Figure 4 .

From Figure 4(a), we can easily see that the parameters $\beta, C_{a}, C_{s}, D_{E_{2}}$ and $D_{I_{1}}$ have significant effect on $\mathscr{R}_{e}$. Furthermore, the effective contact rate $\beta$ and $\mathscr{R}_{e}$ are positively correlated, which means that the increasing of effective contact rate can augment $\mathscr{R}_{e}$. The parameters $C_{a}$ and $C_{s}$ are negatively correlated with $\mathscr{R}_{e}$, which shows that NPIs are strengthened for incubation and infection with infectiousness can help to reduce the infection potential of Omicron variant. The parameters $D_{E_{2}}, D_{I_{1}}$ and $\mathscr{R}_{e}$ are positively correlated, which reflect the peculiarity of Omicron.

Figure 4(b) reveals that $\beta, C_{a}, C_{s}, \theta, D_{E_{2}}, D_{I_{1}}, D_{I_{2}}, \xi$ and $\tau$ effect mainly on the the cumulative infected cases. Logically, the decreasing effective contact rate $\beta$ can decrease the cumulative infected cases, which tell us that an environmental decontamination is an important measures to control the pandemic. For $C_{a}$ and $C_{s}$, we can strengthen NPIs, such as keeping social distance, wearing a well-fitting mask, staying home and isolating from other people for incubation and infection with infectiousness to control Omicron pandemic. Considering $\xi, \tau$ and $\theta$, we suggest that everyone should get vaccinated and boosted as soon as they are eligible, including people who have already had COVID-19. Since $D_{E_{2}}, D_{I_{1}}$ and $D_{I_{2}}$ reflect the peculiarity of Omicron, a drug intervention may be the most effective means.

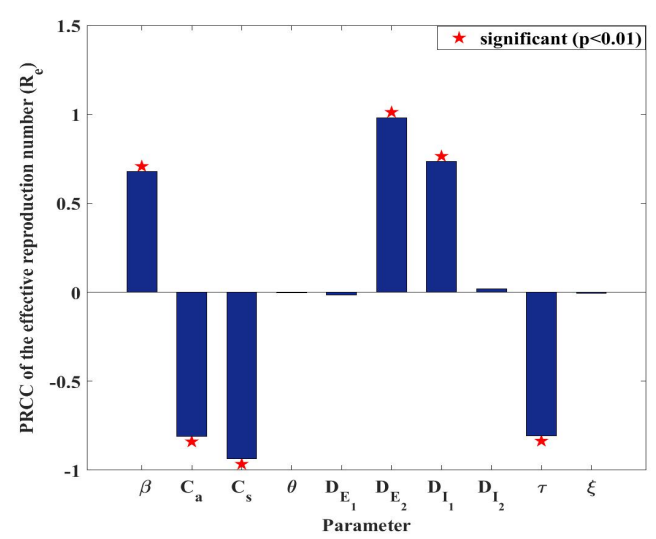

(a)

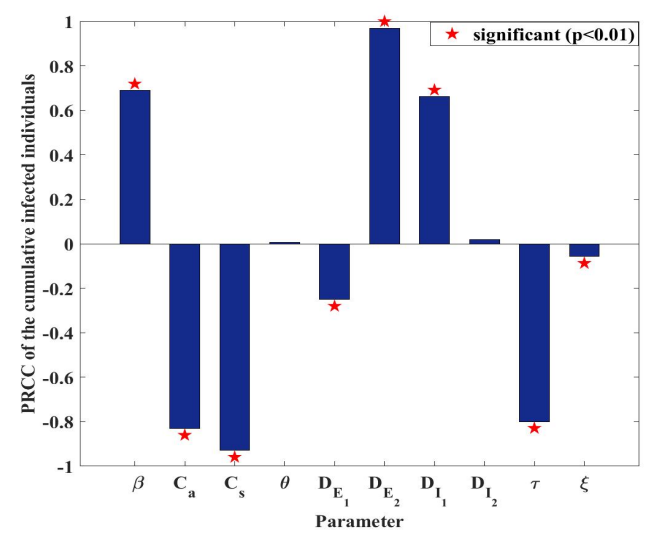

(b)

Figure 4: Sensitivity analysis among parameters, $\mathscr{R}_{e}$ and the total infections cases 
The specific impact of parameters on the effective reproduction number $\mathscr{R}_{e}$ and the cumulative infected individuals is shown in Table 2 .

Table 2: PRCC of the cumulative infected individuals $(C)$ and the effective reproduction number $\left(R_{e}\right)$

\begin{tabular}{ccccc}
\hline \multirow{2}{*}{ Parameter } & \multicolumn{2}{c}{$R_{e}$} & \multicolumn{2}{c}{$C$} \\
\cline { 2 - 5 } & PRCC & p-value & PRCC & p-value \\
\hline$\beta$ & 0.6773 & 0.0000 & 0.6888 & 0.0000 \\
$C_{a}$ & -0.8100 & 0.0000 & -0.8306 & 0.0000 \\
$C_{s}$ & -0.9360 & 0.0000 & -0.9294 & 0.0000 \\
$\theta$ & -0.0031 & 0.7829 & 0.0055 & 0.6243 \\
$D_{E_{1}}$ & -0.0161 & 0.1489 & -0.2503 & 0.0000 \\
$D_{E_{2}}$ & 0.9815 & 0.0000 & 0.9695 & 0.0000 \\
$D_{I_{1}}$ & 0.7344 & 0.0000 & 0.6613 & 0.0000 \\
$D_{I_{2}}$ & 0.0188 & 0.0931 & 0.0186 & 0.0960 \\
$\tau$ & -0.8061 & 0.0000 & -0.7994 & 0.0000 \\
$\xi$ & -0.0073 & 0.5124 & -0.0577 & 0.0000 \\
\hline
\end{tabular}

\subsection{The influence of NPIs and vaccines}

In order to visually reflect the effect of parameters, we increase the negative correlation parameters $C_{a}, C_{s}, \xi, \tau$ to see the change of the cumulative infected (see Figure 5).

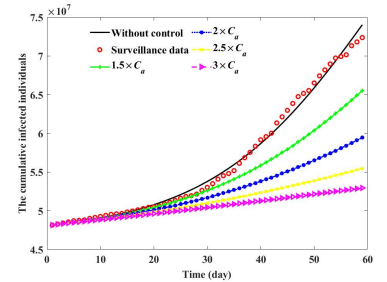

(a)

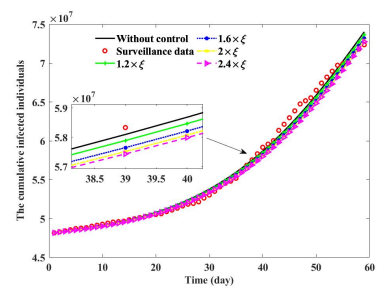

(c)

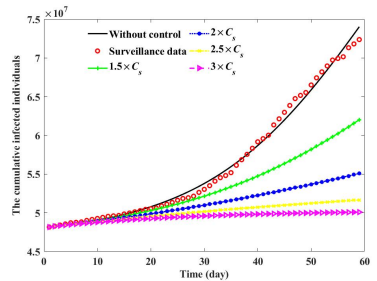

(b)

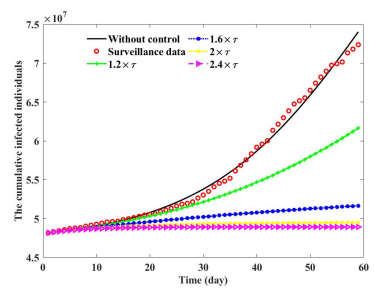

(d)

Figure 5: The influence of $C_{a}, C_{s}, \xi, \tau$ on the cumulative infected individuals

Figure 5 reflects the trend of the impact of key and changeable parameters on cumulative infected individuals. It then follows from Figure $5(a)$ and $(b)$ that the cumulative infected individuals 
decrease with the increase of $C_{a}$ and $C_{s}$, and the decreasing range is relatively large. Figure $5(c)$ and $(d)$ reveal that vaccination remains the best way to protect yourself from COVID-19 and reduce its impact on our communities. Obviously, the efficacy of vaccines has a significant effect on reducing the cumulative infected individuals. Due to the B.1.1.529 (Omicron) variant reducing the efficacy of vaccines and the low vaccination rate it will take months/years until herd immunity is achieved, NPIs play an important role in the prevention and control of COVID-19 pandemic. In the following, we accurately analyze the impact of of Non-pharmaceutical interventions(NPIs) in the controlling of Omicron.

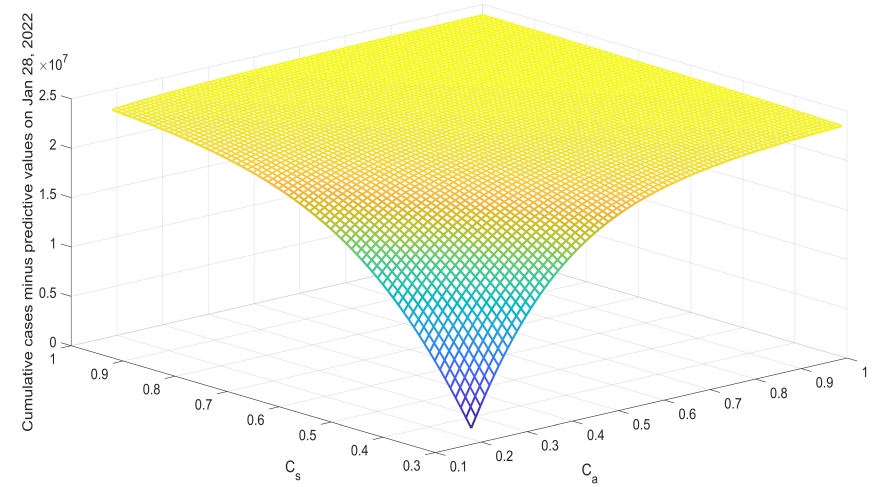

Figure 6: The decrement of numbers of infectious with the increase of $C_{s}$ and $C_{a}$.

Figure 6 shows that $C_{s}$ and $C_{a}$ can effectively reduce the cumulative cases. Let $P$ be the decrement of numbers of infectious with the increase of $C_{s}$ and $C_{a}$ and other parameters remain unchanged. More intuitively, we have listed the specific numbers under the different intensity of NPIs(see Table 3).

Table 3: The relationship between $P$ and $C_{s}$ and $C_{a}$

\begin{tabular}{|l|c|c|c|c|c|c|c|}
\hline$P \backslash C_{a}$ & \multirow{2}{*}{0.25} & 0.30 & 0.35 & 0.40 & 0.45 & 0.50 & 0.55 \\
\cline { 1 - 8 }$C_{s}$ & & & & & & & \\
\hline 0.25 & 2193012 & 6829268 & 10628490 & 13655266 & 16015034 & 17826122 & 19202489 \\
\hline 0.30 & 6278878 & 10204768 & 13339429 & 15785173 & 17662236 & 19087127 & 20162639 \\
\hline 0.35 & 9763993 & 13009947 & 15545466 & 17491289 & 18967047 & 20079078 & 20915447 \\
\hline 0.40 & 12666658 & 15295503 & 17313090 & 18841975 & 19992234 & 20855512 & 21504429 \\
\hline 0.45 & 15034670 & 17127191 & 18711686 & 19901940 & 20793364 & 21461773 & 21965320 \\
\hline 0.50 & 16933247 & 18575890 & 19808021 & 20728878 & 21417641 & 21935110 & 22326802 \\
\hline 0.55 & 18434315 & 19710283 & 20661943 & 21371969 & 21903948 & 22305474 & 22611549 \\
\hline
\end{tabular}

It then follows from the data in Table 3 if $C_{s}$ is raised from 0.30 to 0.50 and $C_{a}$ is raised from 0.17 to 0.35 , then there will be 19,808,021 fewer infected individual. Supposing that $C_{a}$ 
changes from 0.17 to 0.50 and $C_{s}$ is raised from 0.30 to 0.35 , then there will be $20,079,078$ fewer infected individual. If both $C_{a}$ and $C_{s}$ are increased to 0.50 , then there will be 21,935,110 fewer infected individual. According to the disease-induced mortality rate in the United States, if we take $C_{s}=0.35, C_{a}=0.50$, then 35,185 people are saved; If $C_{s}=0.50, C_{a}=0.35$, then 34,710 people are saved; If $C_{s}=0.50$ and $C_{a}=0.50$, then 38,437 people are saved.

\subsection{The relationship among $\mathscr{R}_{e}$, NPIs and vaccine}

At present, the efficacy of vaccine is not relatively low, and someone was diagnosed with the Omicron variant of COVID-19 despite having received two shots of the vaccine. In the following, we assume that all people are vaccined Janssen COVID-19 Vaccine and consider the relationship between $\mathscr{R}_{e}$ and $C_{s}, C_{a}$ (see Figure 7 ).

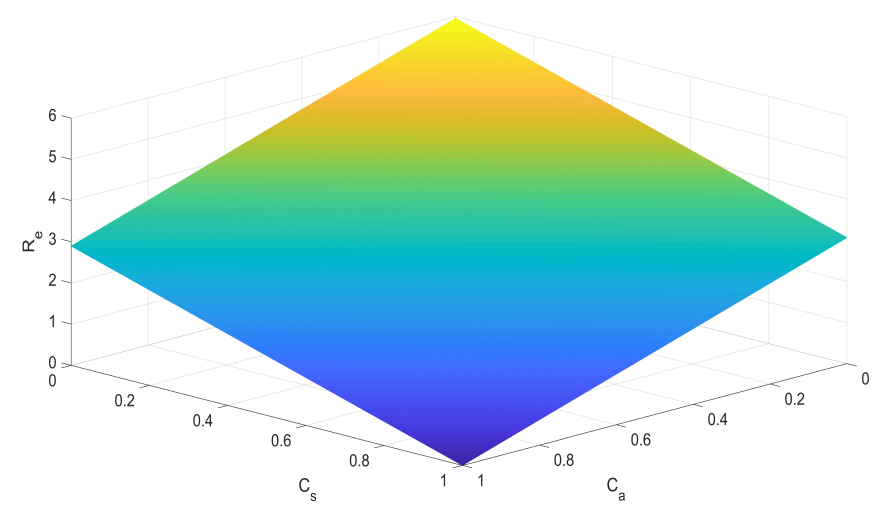

Figure 7: When $\tau=0.33$ and $\xi=1$, the image in three dimensions of relationship among $\mathscr{R}_{e}, C_{s}$ and $C_{a}$.

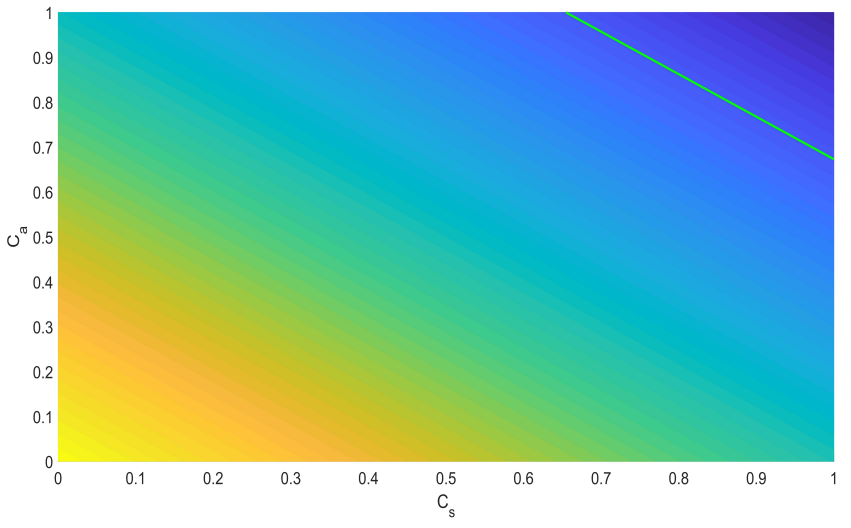

Figure 8: When $\tau=0.33$ and $\xi=1$, the relationship between $C_{s}$ and $C_{a}$. 
It can be seen from the above discussion that NPIs play a very significant role for the disease control. Figure 8 is the projection of Figure 7 on the $C_{s} \times C_{a}$ plane. The green line in Figure 8 represents $\mathscr{R}_{e}=1$. Figure 7 shows if $\left(C_{a}, C_{s}\right)$ belongs the area above the green line, then $\mathscr{R}_{e}<1$, while $\mathscr{R}_{e}>1$ in the area below the green line. Our numerical results shows that NPIs are indispensable even if all the people were vaccinated when the efficiency of vaccine is relatively low. In other words, in order to control the spread of Omicron, NPIs must be strengthened to make $C_{s}$ and $C_{a}$ in the area above the green line even if each people is vaccinated. Particularly, we suggest that NPIs should be strengthened, not weakened in the United States.

In the following, we study the role of the vaccine in the absence of NPIs.

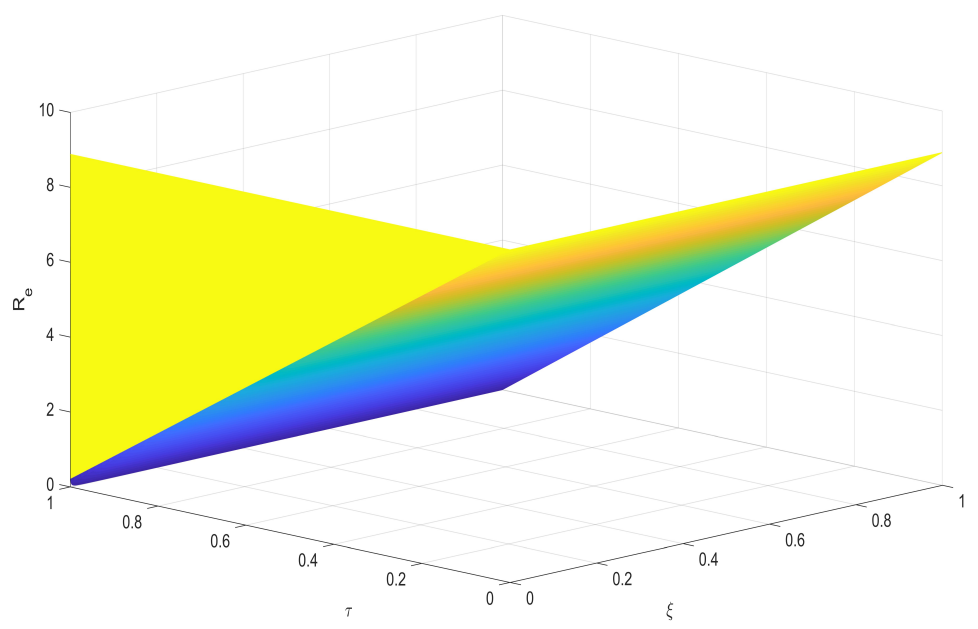

Figure 9: When $C_{s}=0$ and $C_{a}=0$, the relationship between $\xi$ and $\tau$ and $\mathscr{R}_{e}$.

We observe that $\mathscr{R}_{e}$ decreases with the improvement of $\xi$, and finally $\mathscr{R}_{e}$ is less than 1 when $\tau=0.9$ (see Figure 9$)$. $\mathscr{R}_{e}>1$ holds even if $\xi=1$ when $\tau=0.33$. Let $\mathscr{R}_{e}=1, C_{a}=C_{s}=0$ and $\xi=1$, it then follows from (4.1) that $\tau=0.887$. In other words, $\mathscr{R}_{e}>1$ always holds when $\tau<0.887$. Hence, we have gotten a minimum standard of the efficacy rate of vaccine.

\subsection{Herd immunity}

In the following, we look for the possibility of herd immunity in the United States. It is easy see that $\tau \xi$ indicates the proportion of antibody produced after vaccination. Let $C_{a}=C_{s}=\xi=\tau=0$ and $\mathscr{R}_{0}=8.883$. Theoretically, we conclude if $1-\frac{1}{\mathscr{R}_{0}}<\tau \xi$ is satisfied [10], then the herd immunity is formed. 


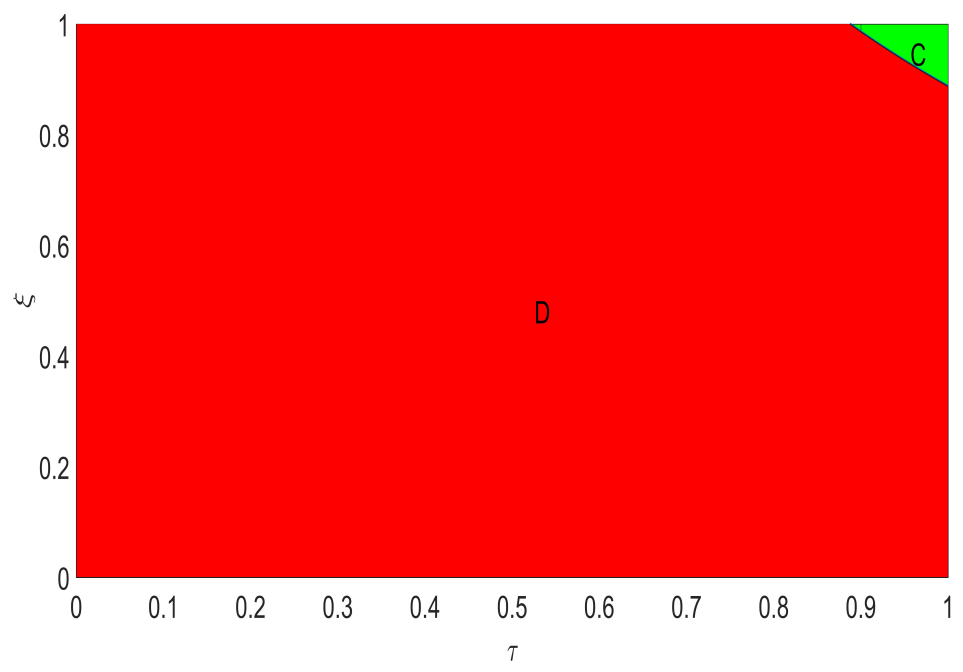

Figure 10: When $C_{a}=0$ and $C_{s}=0$, the relationship between herd immunity and $\tau$ and $\xi$

From Figure 10, it reveals that $\xi=0.887$ when $\tau=1$, and $\tau=0.887$ if $\xi=1$. The intersection of the area $C$ and $D$ is called the herd immunity line which satisfies $\tau \xi=0.887$ and $C$ is the herd immunity area where the condition $\tau \xi>0.887$ is satisfied. Since there is a huge problem that some of American without any face coverings and unwilling to take the vaccine, and the efficacy of vaccines is low against Omicron, everyone should get vaccinated and boosted as soon as they are eligible, including people who have already had COVID-19.

\subsection{Almost periodicity of disease-induced mortality rate}

In the process of parameters analysis, we find that the disease-induced mortality rate demonstrates the periodic oscillation. To further confirm this, we use an almost periodic function

$d(t)=2.687608 \times 10^{-5}+1.419898 \times 10^{-5} \times \cos ((407 / 400) * \sqrt{5} \pi t)+1.317339 \times 10^{-6} \times \sin ((103 / 100) * \sqrt{6} \pi t)$

to simulate the curve by MATLAB, and the simulation result can be shown in Figure 11. It's obviously that the surveillance and simulation fit very well. Since seasonal variations in temperature, rainfall, resource availability, contact rates, the birth and death rates of populations and immune defences are ubiquitous and can exert strong pressures on population dynamics [1], we think that this result is reasonable. 


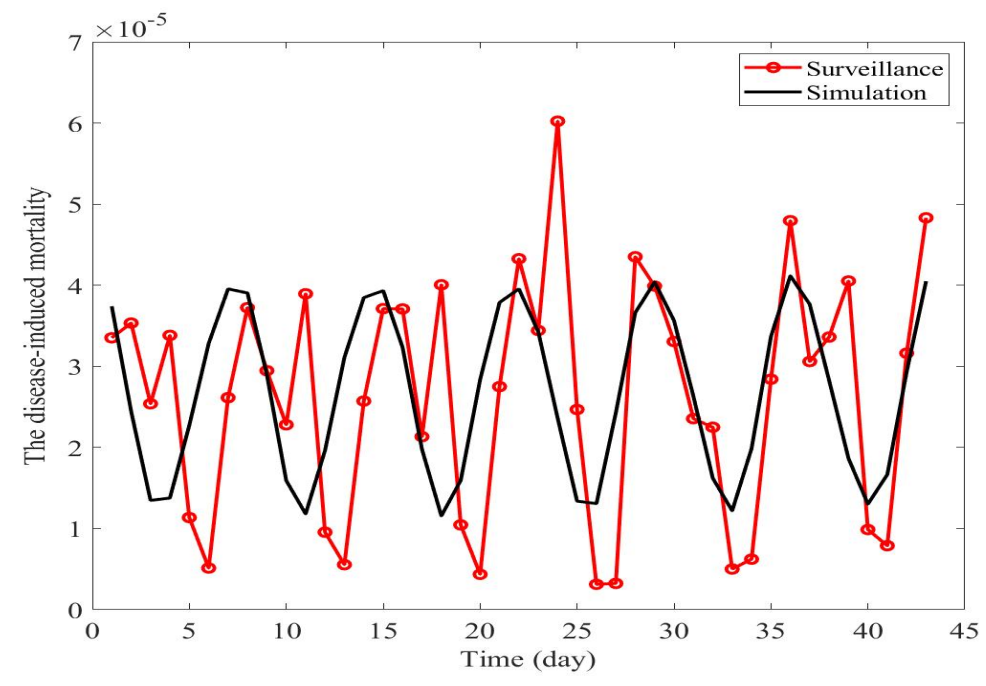

Figure 11: The simulation of the disease-induced mortality

\section{Discussion}

Emphasizing non-pharmaceutical interventions(NPIs) and vaccines, the transmission dynamics of an Omicron variant of COVID-19 model is considered by means of the reproduction number. In section 4, we considered the situations in the United States. Our numerical result predicts that the the United States epidemic will uniformly exist and form an endemic disease if the existing intensity of NPIs and the vaccine efficacy are maintained. If the outbreak occurs repeatedly, we suggest that NPIs should be strengthened. Furthermore, it is shown that NPIs are indispensable even if all the people were vaccinated when the efficiency of vaccine is relatively low. In order to obtain the herd immunity, we speculate in numerical simulation that the minimum efficacy of vaccine is $88.7 \%$. In the face of the crisis of confidence of vaccine and logistical challenges, the herd immunity area is given. Certainly, we expect that COVID-19 will die out as soon as possible by the efforts of people of all over the world.

Author contributions BW contributed to conceptualization, project administration, writingoriginal draft, writing-review and editing; ZW and YW contributed to writing-review and editing, supervision; YX and JZ contributed to formal analysis, software, writing-original draft, writingreview and editing; ZW and ZM contributed to data curation, software.

Funding Information This work was supported by Natural Science Foundation of Gansu Province of China (21JR7RA535, 21JR7RA537) and NSF of China $(11501269,11731005,12071193)$.

Availability of data and material The dataset used and/or analyzed during the current study is available from the published works. 


\section{Declarations}

Conflict of interest The authors declare no conflict of interest.

Ethics approval and consent to participate The study protocol was approved by School of Mathematics and Statistics of Lanzhou University.

\section{Appendix A. Proof of Theorem 3.1 and 3.2}

Proof of Theorem 3.1: It is easy to see that $S(t)$ and $V(t)$ satisfy

$$
\frac{d S}{d t} \leq \Lambda-\xi S-\mu S+\theta R
$$

and

$$
\frac{d V}{d t} \leq \xi S-\mu V
$$

respectively. Since system (3.1) has a unique positive constant solution $\left(S^{*}, V^{*}\right)$, which is global asymptotically stable, by the comparsion theorem, for any $x^{0} \in \mathbb{R}_{+}^{7}$ and $\varepsilon>0$, there exists $t_{0}>0$ such that

$$
S(t) \leq S^{*}+\varepsilon, V(t) \leq V^{*}+\varepsilon, \quad \forall t \geq t_{0} .
$$

It then follows that

$$
\begin{aligned}
\frac{d E_{1}}{d t} \leq & \beta\left(1-C_{a}\right) \frac{\left(S^{*}+\epsilon\right) E_{2}}{N}+\beta\left(1-C_{s}\right) \frac{\left(S^{*}+\epsilon\right) I_{1}}{N}+\beta\left(1-C_{a}\right)(1-\tau) \frac{\left(V^{*}+\epsilon\right) E_{2}}{N} \\
& +\beta\left(1-C_{s}\right)(1-\tau) \frac{\left(V^{*}+\epsilon\right) I_{1}}{N}-\frac{E_{1}}{D_{E_{1}}}-\mu E_{1}, \\
\frac{d E_{2}}{d t} \leq & \frac{E_{1}}{D_{E_{1}}}-\frac{E_{2}}{D_{E_{2}}}-\mu E_{2}, \\
\frac{d I_{1}}{d t} \leq & \frac{E_{2}}{D_{E_{2}}}-\frac{I_{1}}{D_{I_{1}}}-\mu I_{1} .
\end{aligned}
$$

we consider the following system

$$
\begin{aligned}
\frac{d E_{1}}{d t}= & \beta\left(1-C_{a}\right) \frac{\left(S^{*}+\epsilon\right) E_{2}}{N}+\beta\left(1-C_{s}\right) \frac{\left(S^{*}+\epsilon\right) I_{1}}{N}+\beta\left(1-C_{a}\right)(1-\tau) \frac{\left(V^{*}+\epsilon\right) E_{2}}{N} \\
& +\beta\left(1-C_{s}\right)(1-\tau) \frac{\left(V^{*}+\epsilon\right) I_{1}}{N}-\frac{E_{1}}{D_{E_{1}}}-\mu E_{1}, \\
\frac{d E_{2}}{d t}= & \frac{E_{1}}{D_{E_{1}}}-\frac{E_{2}}{D_{E_{2}}}-\mu E_{2}, \\
\frac{d I_{1}}{d t}= & \frac{E_{2}}{D_{E_{2}}}-\frac{I_{1}}{D_{I_{1}}}-\mu I_{1} .
\end{aligned}
$$

Denote $F_{1}^{\epsilon}=\beta\left(1-C_{a}\right) \frac{\left(N^{*}+2 \epsilon\right)-\tau\left(V^{*}+\epsilon\right)}{N}$ and $F_{2}^{\epsilon}=\beta\left(1-C_{s}\right) \frac{\left(N^{*}+2 \epsilon\right)-\tau\left(V^{*}+\epsilon\right)}{N}$.

Let

$$
Y^{\epsilon}=\left(\begin{array}{ccc}
0 & F_{1}^{\epsilon} & F_{2}^{\epsilon} \\
0 & 0 & 0 \\
0 & 0 & 0
\end{array}\right) .
$$

Since $\mathscr{R}_{e}<1$, then $\omega(-Z+Y)<0$, where $\omega(-Z+Y)$ is the exponential growth bound [40]. By the continuity of spectral bound, there exists a sufficiently small $\epsilon_{1}>0$ such that $\omega\left(-Z+Y^{\epsilon}\right)<0$ 
for $0<\epsilon<\epsilon_{1}$, which implies that the trivial solution of the system (1) is globally asymptotically stable. By the comparison theorem of ordinary differential equation, we deduce that $E_{1} \rightarrow 0, E_{2} \rightarrow$ $0, I_{1} \rightarrow 0$ as $t \rightarrow \infty$. It then follows that system (3.1) is the limiting system of $S, V$ equation in system (2.1). We also could get that $I_{2}, R$ equation admit the limiting system

$$
\begin{aligned}
& \frac{d I_{2}}{d t}=-\frac{I_{2}}{D_{I_{2}}}-\mu I_{2}, \\
& \frac{d R}{d t}=\frac{I_{2}}{D_{I_{2}}}-\mu R-\theta R .
\end{aligned}
$$

It is easy to see that the solutions in (2) convergence to $(0,0)$. Finally, by the theory of asymptotically autonomous systems (see, e.g. [4] ), we conclude that the solution of system (2.1) converges to $\left(S^{*}, V^{*}, 0,0,0,0,0\right)$. This confirms the global attractivity of $\mathcal{E}^{*}$ for system $(2.1)$ under the condition $\mathscr{R}_{e}<1$, and hence completes the proof.

Proof of Theorem 3.2: Define

$$
\begin{gathered}
X=\mathbb{R}_{+}^{7}, \\
X_{0}:=\left\{\left(S, V, E_{1}, E_{2}, I_{1}, I_{2}, R\right) \in X: E_{1}>0, E_{2}>0, I_{1}>0\right\} \\
\partial X_{0}:=X \backslash X_{0}
\end{gathered}
$$

Then $X_{0}$ and $\partial X_{0}$ are relatively open and closed in $\mathbb{R}^{7}$, respectively. For any $x^{0} \in X_{0}$, let $\psi_{t}\left(x^{0}\right)$ be the unique solution of system (2.1) with initial data $x^{0}$. It is easy to see that $X_{0}$ is a positively invariant set. According to the arguments in Section 2, the solution of (2.1) is ultimately bounded in $X$, which implies that $\psi_{t}: X \rightarrow X$ is point dissipative on $X$. It follows from [15, Theorem 3.4.8] that $\psi_{t}$ has a global compact attractor $\mathcal{A}$.

Define

$$
M_{\partial}:=\left\{x^{0} \in \partial X_{0}: \psi_{t}\left(x^{0}\right) \in \partial X_{0}, \forall t \geq 0\right\}
$$

and

$$
\mathcal{M}:=\left\{x^{0} \in X: x_{1}^{0}=S^{*}, x_{2}^{0}=V^{*}, x_{3}^{0}=x_{4}^{0}=x_{5}^{0}=0\right\} .
$$

We now show that

$$
M_{\partial}=\mathcal{M}
$$

For any $x^{0} \in \mathcal{M}$, the solution $\psi_{t}\left(x^{0}\right)$ satisfies $E_{1}\left(t, x^{0}\right)=0, E_{2}\left(t, x^{0}\right)=0, I_{1}\left(t, x^{0}\right)=0$ for all $t \geq 0$. Hence, $x^{0} \in M_{\partial}$ and $\mathcal{M} \subset M_{\partial}$.

For any $x^{0} \in \partial X_{0} \backslash \mathcal{M}$, there is $\left(x_{3}^{0}, x_{4}^{0}, x_{5}^{0}\right)$ such that $\left(x_{3}^{0}, x_{4}^{0}, x_{5}^{0}\right)=\left(E_{1}(0), E_{2}(0), I_{1}(0)\right)>$ $(0,0,0)$.

Case 1 Let $E_{1}(0)>0$. Then the third equation of system (2.1) satisfies

$$
\frac{d E_{1}}{d t} \geq-\frac{E_{1}}{D_{E_{1}}}-\mu E_{1} .
$$

Furthermore, there exists a $t_{0}>0$ such that $E_{1}(t)>0$ for all $t \geq t_{0}$.

From the third equation of system (2.1), we can get $E_{2}(t)>0 \forall t \geq t_{0}+1$. Then, from the fourth equation of system (2.1), we deduct that $I_{1}(t)>0$ for all $t \geq t_{0}+2$. 
Case 2 Let $E_{2}(0)>0$. By the fourth equation of system (2.1), we have

$$
\frac{d E_{2}}{d t} \geq-\frac{E_{2}}{D_{E_{2}}}-\mu E_{2}
$$

Thus, we can get $E_{2}(t)>0, \forall t>0$. Now, the third equation satisfies

$$
\frac{d E_{1}}{d t} \geq \beta\left(1-C_{a}\right) \frac{S E_{2}}{N}-\frac{E_{1}}{D_{E_{1}}}-\mu E_{1} .
$$

It is easy to see that $E_{1}(t)>0$ for $t>1$. By the arguments in Case 1, we can obtain that $\left(E_{1}(t), E_{2}(t), I_{1}(t)\right) \gg(0, \cdots, 0,0 \cdots, 0,0, \cdots, 0)$ for all $t>t_{0}+3$.

Case 3 Let $I_{1}(0)>0$. By the fifth equation of system (2.1), we have

$$
\frac{d I_{1}}{d t} \geq-\frac{I_{1}}{D_{I_{1}}}-\mu I_{1} .
$$

Hence, we can get $I_{1}(t)>0, \forall t>0$. Now, the second equation of system (2.1) satisfies

$$
\frac{d E_{1}}{d t} \geq \beta\left(1-C_{s}\right) \frac{S I_{1}}{N}-\frac{E_{1}}{D_{E_{1}}}-\mu E_{1} .
$$

It is easy to see that $E_{1}(t)>0$ for $t>1$. By the arguments in Case 1, we can obtain that $\left(E_{1}(t), E_{2}(t), I_{1}(t)\right) \gg(0, \cdots, 0,0 \cdots, 0,0, \cdots, 0)$ for all $t>t_{0}+3$.

Then $M_{\partial} \subset \mathcal{M}$. Hence, $M_{\partial}=\mathcal{M}$.

We claim that $W^{s}(\mathcal{M}) \cap X_{0}=\emptyset$, where $W^{s}(\mathcal{M})$ is the stable manifold of $\mathcal{M}$. Let $\bar{\lambda}=$ $\beta\left(1-C_{a}\right) \frac{\left(N^{*}-2 \epsilon\right)-\tau\left(V^{*}-\epsilon\right)}{N}$ and $\bar{\eta}=\beta\left(1-C_{s}\right) \frac{\left(N^{*}-2 \epsilon\right)-\tau\left(V^{*}-\epsilon\right)}{N}$. Denote

$$
\bar{F}_{1}^{\epsilon}=\bar{\lambda}, \bar{F}_{2}^{\epsilon}=\bar{\eta}
$$

Let

$$
\bar{Y}^{\epsilon}=\left(\begin{array}{ccc}
0 & F_{1}^{\epsilon} & F_{2}^{\epsilon} \\
0 & 0 & 0 \\
0 & 0 & 0
\end{array}\right) .
$$

Since $\mathscr{R}_{e}>1$, then $\omega(Y-Z)>0$. By the continuity of spectral bound, there exists a sufficiently small $\epsilon_{1}>0$ such that $\omega\left(\bar{Y}_{\epsilon}-Z\right)>0$ for $0<\epsilon \leq \epsilon_{1}$.

Claim. If $x^{0} \in X_{0}$, then

$$
\limsup _{t \rightarrow \infty} d\left(\psi_{t}\left(x^{0}\right), \mathcal{M}\right) \geq \epsilon_{1}
$$

On the contrary, we assume that there exists $\bar{x}^{0} \in X_{0}$ such that $\limsup _{t \rightarrow \infty} d\left(\psi_{t}\left(\bar{x}^{0}\right), \mathcal{M}\right)<\varepsilon_{1}$. It then follows that there exists $t_{0}>0$ such that

$$
S^{*}-\varepsilon_{1}<S(t)<S^{*}+\varepsilon_{1}, V^{*}-\varepsilon_{1}<V(t)<V^{*}+\varepsilon_{1}
$$

for all $t \geq t_{0}$. Hence, we have

$$
\begin{aligned}
\frac{d E_{1}}{d t} \geq & \beta\left(1-C_{a}\right) \frac{\left(S^{*}-\varepsilon_{1}\right) E_{2}}{N}+\beta\left(1-C_{s}\right) \frac{\left(S^{*}-\varepsilon_{1}\right) I_{1}}{N}+\beta\left(1-C_{a}\right)(1-\tau) \frac{\left(V^{*}-\varepsilon_{1}\right) E_{2}}{N} \\
& +\beta\left(1-C_{s}\right)(1-\tau) \frac{\left(V^{*}-\varepsilon_{1}\right) I_{1}}{N}-\frac{E_{1}}{D_{E_{1}}}-\mu E_{1}, \\
\frac{d E_{2}}{d t} \geq & \frac{E_{1}}{D_{E_{1}}}-\frac{E_{2}}{D_{E_{2}}}-\mu E_{2}, \\
\frac{d I_{1}}{d t} \geq & \frac{E_{2}}{D_{E_{2}}}-\frac{I_{1}}{D_{I_{1}}}-\mu I_{1} .
\end{aligned}
$$


Since $-Z+\bar{Y}_{\epsilon}$ is irreducible and essentially nonnegative, it has a positive eigenvector associated with $\omega\left(-Z+\bar{Y}_{\epsilon}\right)>0$. By the comparison theorem of ordinary differential equations, we have $\lim _{t \rightarrow \infty} E_{1}(t)=\infty, \lim _{t \rightarrow \infty} E_{2}(t)=\infty, \lim _{t \rightarrow \infty} I_{1}(t)=\infty$, a contradiction. The claim is proved.

The set $M_{\partial}=\mathcal{M}$ is an isolated invariant set and acyclic. By [32, Theorem 4.6], we conclude that system (2.1) is uniformly persistent in $X_{0}$ whenever $\mathscr{R}_{e}>1$. That is, there is a $\tilde{\varepsilon}>0$ such that

$$
\liminf _{t \rightarrow \infty} E_{1}(t)>\tilde{\varepsilon}, \liminf _{t \rightarrow \infty} E_{2}(t)>\tilde{\varepsilon}, \liminf _{t \rightarrow \infty} I_{1}(t)>\tilde{\varepsilon} .
$$

This completes the proof.

\section{References}

[1] S. Altizer, A. Dobson, P. Hosseini, P. Hudson, M. Pascual, P. Rohani, Seasonality and the dynamics of infectious diseases, Ecology Letters, 9(2006), 467-484.

[2] M. A. Acuña-Zegarraa, S. Díaz-Infante, D. Baca-Carrasco, D. Olmos-Liceaga, COVID-19 optimal vaccination policies: A modeling study on efficacy, natural and vaccine-induced immunity responses, Mathematical Biosciences, 337(2021), 108614.

[3] K. M. Bubar, K. Reinholt, S. M. Kisslermarc, M. Lipsitch, S. Cobey, Y. H. Grad, D. B. Larremore, Model-informed COVID-19 vaccine prioritization strategies by age and serostatus, Science, 371(2021), 916-921.

[4] C. Castillo-Chaves, H. R. Thieme, Asymptotically autonomous epidemic models. In: Arino $\mathrm{O}$ et al (eds) Mathematical population dynamics: analysis of heterogeneity, I. Theory of epidemics. Wuerz, Winnipeg, 33-50.

[5] S. Collie, H. Chemaitelly, A. A Butt, Effectiveness of BNT162b2 Vaccine against Omicron Variant in South Africa, The New England Journal of Medicine, 2021, DOI:10.1056/NEJMc2119270.

[6] Guidelines for the implementation of non-pharmaceutical interventions against COVID-19. Eur. Cent. Dis. Prev. Control (2020); www.ecdc.europa.eu/en/publications-data/covid-19guidelines-non-pharmaceutical-interventions.

[7] J. Cuevas-Maraver, P. G. Kevrekidis, Q. Y. Chen, G. A. Kevrekidis, Víctor Villalobos-Daniel, Z. Rapti, Y. Drossinos, Lockdown measures and their impact on single- and two-age-structured epidemic model for the COVID-19 outbreak in Mexico, Mathematical Biosciences, 336(2021), 108590.

[8] Y. Cheng, N. Ma, C. Witt, S. Rapp, P. S. Wild, M. O. Andreae, U. P0̈schl, H. $\mathrm{Su}$, Face masks effectively limit the probability of SARS-CoV-2 transmission, Science, DOI:10.1126/science.abg6296, (2021).

[9] P. van den Driessche, J. Watmough, Reproduction numbers and sub-threshold endemic equilibria for compartmental models of disease transmission, Math. Biosci., 180(2002), 29-48.

[10] K. Dietz, Transmission and control of arbovirus diseases, D. Ludwig, K. L. Cooke, Epidemiology, society for industrial and applied mathematics (SIAM), 1975, 104-121.

[11] D. Gao, S. Ruan, An SIS patch model with variable transmission coefficients, Math. Biosci., 232(2011), 110-115.

[12] S. Gao, M. Martcheva, H. Miao, L. Rong, A two-sex model of human papillomavirus infection: Vaccination strategies and a case study, Journal of Theoretical Biology 536(2022), 111006.

[13] X. Gao, K. Zhu, B. Qin, V. Olieric, M. Wang, S. Cui, Crystal structure of SARS-CoV2 Orf9b in complex with human TOM70 suggests unusual virus-host interactions, Nature Communications 12(2021), 2843. 
[14] S. Grundel, S. Heyder, T. Hotz, T. K. S. Ritschel, P. Sauerteig, K. Worthmann, How to Coordinate Vaccination and Social Distancing to Mitigate SARS-CoV-2 Outbreaks, SIAM J. APPLIED DYNAMICAL SYSTEMS, 20(2021), 1135-1157.

[15] J. K. Hale, Asymptotic behavior of dissipative systems. American Mathematical Society, Providence, 1988.

[16] P. Hunter, COVID-19 Vaccines Are Probably Less Effective at Preventing Transmission Than Symptoms - Here's Why, 2021, https://theconversation.com/covid-19-vaccines-are-probablyless-effective-at preventing-transmission-than-symptoms-heres-why-156611, accessed March 19, 2021.

[17] S. Huang, W. Lin, J. Li, M. Z. Xin, Y. Wang, Y. Hao, Z. Wang, Q. Deng, B. G. Wang, Transmission Dynamics and High Infectiousness of Coronavirus Disease 2019, Communications on Pure and Applied Analysis,DOI:10.3934/cpaa.2021155.

[18] International Monetary Fund, Policy Responses to COVID-19, 2020, https://www.imf.org/en/ Topics/imf-and-covid19/Policy-Responses-to-COVID-19, accessed October 26, 2020.

[19] International Monetary Fund, World Economic Outlook: A Long and Difficult Ascent, 2020, https:// www.imf.org/en/Publications/WEO/Issues/2020/04/14/weo-april-2020, accessed October 26, 2020.

[20] Q. Jing, M. J. Liu, Z. B. Zhang, L. Q. Fang, J. Yuan, A. R. Zhang, N. E. Dean, L. Luo, M. M. Ma, I. Longini, E. K. ScD, Y. Lu, Y. Ma, N. Jalali, Z. C. Yang, Y. Yang, Household secondary attack rate of COVID-19 and associated determinants in Guangzhou, China: a retrospective cohort study, Lancet Infect. Dis. 20(2020), 1141-1150.

[21] JKK. Asamoah, Z. Jin, G. Sun, B. Seidu, E. Yankson, A. Abidemi, F. T. Oduro, S. E. Moore, E. Okyere, Sensitivity assessment and optimal economic evaluation of a new COVID-19 compartmental epidemic model with control interventions, Chaos Solitons Fractals, 146(2021), 110885.

[22] Q. Li, M. Med, X. Guan, P. Wu, et al., Early transmission dynamics in Wuhan, China, of novel coronavirus-infected pneumonia, N. Engl. J. Med. 382(2020), 1199-1207.

[23] O. Diekmann, J. A. P. Heesterbeek, J. A. J. Metz, On the definition and the computation of the basic reproduction ratio $R_{0}$ in the models for infectious disease in heterogeneous populations, J. Math. Biol., 28(1990),365-382.

[24] Organisation for Economic Co-operation and Development, The Territorial Impact of COVID- 19: Managing the Crisis Across Levels of Government, 2020, http://www.oecd.org/coronavirus/ policy-responses/the-territorial-impact-of-covid-19managing-the-crisis-across-levels-of-government-d3e314e1, accessed October 26, 2020.

[25] A. Park, Yes, We Have COVID-19 Vaccines That Are 95\% Effective. But That Doesn't Mean the End of the Pandemic Is Near, Time, 2020, https://time.com/5913113/covid-19-vaccine, accessed December 14, 2020.

[26] M. Pavelka, K. Van-Zandvoort, S. Abbott, et al., The impact of population-wide rapid antigen testing on SARS-CoV-2 prevalence in Slovakia, Science 372(2021), 635-641.

[27] P. Poletti, M. Tirani, D. Cereda, F. Trentini, G. Guzzetta, G. Sabatino, V. Marziano, A. Castrofino, F. Grosso, G. D. Castillo, R. Piccarreta, A. Andreassi, A. Melegaro, M. Gramegna, M. Ajelli, S. Merler, Association of Age With Likelihood of Developing Symptoms and Critical Disease Among Close Contacts Exposed to Patients With Confirmed SARS-CoV-2 Infection in Italy. JAMA Netw, Open 4, e211085 (2021).

[28] M. Pollán et al. Prevalence of SARS-CoV-2 in Spain (ENE-COVID): a nationwide, populationbased seroepidemiological study, Lancet 396(2020), 535-544.

[29] JRC. Pulliam , C. van Schalkwyk , N. Govender , et al. Increased risk of SARS-CoV-2 reinfection associated with emergence of the Omicron variant in South Africa. December 2, 
2021 (https://www.medrxiv.org/content/10.1101/2021.11.11.21266068v2. opens in new tab). preprint.

[30] J Read, J. Bridgen, D. Cummings et al. Novel coronavirus 2019-nCoV: early estimation of epidemiological parameters and epidemic predictions, 2020, DOI: org/10.1101/2020.01. 2320018549.

[31] S. Riley, K. E. C. Ainslie, O. Eales, C. E. Walters, H. Wang, C. Atchison, C. Fronterre, P. J. Diggle, D. Ashby, C. A. Donnelly, G. Cooke, W. Barclay, H. Ward, A. Darzi, P. Elliott, Resurgence of SARS-CoV-2: Detection by community viral surveillance, Science 372(2021), 990-995.

[32] H, R, Thieme, Persistence under relaxed point-dissipativity (with application to an endemic model), SIAM J Math Anal, 24(1993), 407-435.

[33] I. P. Sinha, R. Harwood, M. GSemple, D. B. Hawcutt, R. Thursfield, O. Narayan, S. E. Kenny, R. Viner, S. L. Hewer, K. W. Southern, COVID-19 infection in children, Lancet Respir. Med., 8(2020), 446-447.

[34] A. Senapati, S. Rana, T. Das, J. Chattopadhyay,Impact of intervention on the spread of COVID-19 in India: A model based study, Journal of Theoretical Biology, 523(2021), 110711.

[35] C. M. Saad-Roy, S. E. Morris, C. J. E. Metcalf, M. J. Mina, R. E. Baker, J. Farrar, E. C. Holmes, O. G. Pybus, A. L. Graham, S. A. Levin, B. T. Grenfell, C. E. Wagner, Epidemiological and evolutionary considerations of SARS-CoV-2 vaccine dosing regimes, Science, DOI:10.1126/science.abg8663 (2021).

[36] B. Tang, X. Wang, Q. Li, N. L. Bragazzi, S. Tang, Y. Xiao, J. Wu, Estimation of the transmission risk of 2019-nCov and its implication for public health interventions, Journal of Clinical Medicine, 2020,9(2):462, DOI:10.3390/jcm9020462.

[37] H. Tian, Y. Liu, Y. Li, C. H. Wu, B. Chen, M. U. G. Karemer, B. Li, J. Cai, B. Xu, Q. Yang, B. Wang, X, Yang, Y. Cui, Y. Song, P. Zheng, Q. Wang, O. N. Bjornstad, R. Yang, B. T. Grenfell, O. G. Pybus, C. Dye, An investigation of transmission control measures during the first 50 days of the COVID-19 epidemic in China, Science, 368(2020), 638-642.

[38] W. Van Lancker, Z. Parolin, COVID-19, school closures, and child poverty: a social crisis in the making, Lancet Public Health 5, e243-e244 (2020).

[39] B. G. Wang, S. Huang, Y. Xiong, M. Z. Xin, J. Li, J. Zhang, Z. Ma, Transmission dynamics of COVID-19 pandemic non-pharmaceutical interventions and vaccination. Journal of Mathematical Biology, arXiv:submit/3826075 [math.DS] 6 Jul 2021.

[40] B.-G. Wang, X.-Q. Zhao, Basic reproduction ratios for almost periodic compartmental epidemic models, J Dyn Diff Equ, 25(2013), 535-562.

[41] J. Wu, K. Leung, M. Bushman, N. Kishore, R. Niehus, P. M. de Salazar, B. J. Cowling, M. Lipsitch, G. M. Leung, Estimating clinical severity of COVID-19 from the transmission dynamics in Wuhan, China. Nat. Med. 26(2020), 506-510.

[42] J. T. Wu, K. Leung, G. M. Leung, Nowcasting and forecasting the potential domestic and international spread of the 2019-nCoV outbreak originating in Wuhan, China: a modelling study. The Lancet, 395(2020), 689-697.

[43] J. Zhang, M. Litvinova, Y. Liang, Y. Wang, W. Wang, S. Zhao, Q. Wu, S. Merler, C. Viboud, A. Vespignani, M. Ajelli, H. Yu, Changes in contact patterns shape the dynamics of the COVID- 19 outbreak in China. Science, 368(2020), 1481-1486.

[44] Y. Zhou, M. Huang, Y. Jiang and X. Zou, Data-Driven Mathematical Modeling and Dynamical Analysis for SARS-CoV-2 Coinfection with Bacteria, Internat. J. Bifur. Chaos Appl. Sci. Engrg., 31(2021), 2150163.

[45] Y. Zou, W. Yang, J. Lai, J. Hou, W. Lin, Vaccination and Quarantine Effect on COVID19 Transmission Dynamics Incorporating Chinese-Spring-Festival Travel Rush: Modeling and Simulations. Bull. Math. Biol. 84(2022), Paper No. 30. 
[46] L. Zhou, X. Rong, M. Fan, L. Yang, H. Chu, L. Xue, G. Hu, S. Liu, Z. Zeng, M. Chen, W. Sun, J. Liu, Y. Liu, S. Wang, H. Zhu, Modeling and Evaluation of the Joint Prevention and Control Mechanism for Curbing COVID-19 in Wuhan. Bull. Math. Biol., 84(2022), Paper No. 28.

[47] World Health Organization, WHO Coronavirus disease (COVID-19) Dashboard, URL: https://covid19.who.int/.

[48] World Health Organization, Coronavirus disease (COVID-19) pandemic, URL: https://www.who.int/en/activities/tracking-SARS-CoV-2-variants/.

[49] https://covid19.who.int/region/amro/country/us.

[50] https: //ourworldindata.org/covid-vaccinations 